SLAC-PUB-12119

September 2006

\title{
PROSPECTS OF HIGH ENERGY LABORATORY ASTROPHYSICS *
}

\author{
JOHNNY S.T. NG \\ Stanford Linear Accelerator Center \\ Stanford, California 94309, USA \\ jng@SLAC.Stanford.edu \\ PISIN CHEN \\ Stanford Linear Accelerator Center \\ Stanford, California 94309, USA \\ chen@SLAC.Stanford.edu
}

\begin{abstract}
Ultra high energy cosmic rays (UHECR) have been observed but their sources and production mechanisms are yet to be understood. We envision a laboratory astrophysics program that will contribute to the understanding of cosmic accelerators with efforts to: 1) test and calibrate UHECR observational techniques, and 2) elucidate the underlying physics of cosmic acceleration through laboratory experiments and computer simulations. Innovative experiments belonging to the first category have already been done at the SLAC FFTB. Results on air fluorescence yields from the FLASH experiment are reviewed. Proposed future accelerator facilities can provided unprecedented highenergy-densities in a regime relevant to cosmic acceleration studies and accessible in a terrestrial environment for the first time. We review recent simulation studies of nonlinear plasma dynamics that could give rise to cosmic acceleration, and discuss prospects for experimental investigation of the underlying mechanisms.
\end{abstract}

Keywords: laboratory astrophysics; cosmic accelerator; ultra high energy cosmic rays.

\section{Introduction}

The field of laboratory astrophysics, terrestrial investigations designed to understand physical phenomenon associated with astrophysical systems, is very broad and has a long history. For example, a detailed understanding of atomic and molecular phenomena has been critical to the quantitative interpretation of the spectra of astronomical objects. More recently, advances in the energy and intensities of lasers and particle accelerators promise to produce high energy density conditions approaching those in extreme astrophysical environments associated with supernova explosions and gamma-ray bursts. ${ }^{1}$ Many aspects of these extreme phenomena can be investigated using very high intensity photon and particle beams. ${ }^{2,3}$

*Work supported by U.S. Department of Energy under contract No. DE-AC02-76SF00515. 
Our high energy laboratory astrophysics program consists of efforts to 1) test and calibrate UHECR detection techniques and 2) elucidate acceleration principles that operate on the astronomical scale through laboratory experiments and computer simulations. Innovative experiments belonging to the first category have already been performed at the SLAC FFTB. These include the first observation of radio Cherenkov signal induced by the Askaryan effect, ${ }^{4}$ the FLASH experiment using particle beams to calibrate fluorescence-based techniques for observing ultra high energy cosmic rays, ${ }^{5,6,7}$ and more recently, a beam test experiment using a 6.5 ton ice target to produce realistic Askaryan radio impulse signal to calibrate the ANITA payload. ${ }^{8}$ In this paper, we review results on fluorescence yield measurements from the FLASH experiment. We also discuss simulation studies of non-linear plasma dynamics that could be responsible for the acceleration of these cosmic particles and prospects for future experiments.

An UHECR generates a shower of secondary particles as it enters the earth's atmosphere. The shower in turn excites atmospheric molecules, producing fluorescent light. The primary cosmic ray's energy can be determined by measuring the luminosity of this fluorescence. The energy scale, a major source of the systematic uncertainty in the UHECR's energy, depends on our knowledge of the absolute fluorescence yield. In Section 2, we review results on precision fluorescence yield measurements at the SLAC Final Focus Test Beam facility.

It is generally believed that the interaction of astrophysical relativistic outflows with ambient plasma gives rise to particle acceleration. In particular, kinetic energy of the outflow could be converted into plasma instabilities, which, in turn, power particle acceleration. In Section 3, we review recent simulation studies aimed to understand the underlying acceleration mechanisms.

Future experiments to test cosmic acceleration models will benefit from recent advances in accelerator technology. The proposed SABER facility at SLAC will provide unprecedented high intensity electron and positron beams. ${ }^{9}$ The prospects of future laboratory experiments at SABER are discussed in Section 4.

\section{Test and Calibration of UHECR Detection Methods}

\subsection{Results of the FLASH Experiment}

There are two techniques commonly used to measure the energy of ultra high energy cosmic rays. The ground array technique uses a large array of detectors to count the number of shower charge particles reaching the ground level; the larger the count the higher the primary energy. A complementary technique uses a system of mirrors and photomultipliers to image the fluorescence light produced in the shower. The total light yield is proportional to the energy. Two experiments with similar accumulated exposure, ground-array AGASA ${ }^{10}$ and fluorescence-based HiRes ${ }^{11}$, reported apparent discrepancies in observed flux at energy above $4 \times 10^{18} \mathrm{eV}$ and in the energy at which the power-law spectrum changes shape. ${ }^{12}$ Both experiments are making efforts to improve their understanding of the systematics of energy measurements. 
The measurement of cosmic rays in this energy regime is of particular importance to understanding their properties. In particular, because of interaction with the cosmic microwave background, the flux of cosmic rays above the so-called GreisenZatsepin-Kuzmin (GZK) cut-off is expected to reduce significantly. ${ }^{13}$ The cut-off is at $\sim 10^{19} \mathrm{eV}$ for cosmic protons originating from a distance larger than $50 \mathrm{Mpc}$. A significant excess above this energy would indicate new physics. Thus, efforts to further improve the understanding of these detection techniques are needed.

The SLAC E-165 experiment - Fluorescence in Air from Showers (FLASH) was carried out to address unresolved issues in air fluorescence: the detailed shape of the fluorescence spectrum, the pressure and atmospheric impurities dependence, and the dependence of fluorescence yield on shower particle energy. While previous works on air fluorescence have been instrumental for existing UHECR measurements, improvement in the level of accuracy and confidence is necessary. Several other groups are also working on these issues. ${ }^{14}$ The FLASH experiment has now been completed. Its findings are reported below.

\subsubsection{Pressure Dependence of Air Fluorescence Yield}

The cosmic ray shower is initiated high in the atmosphere, with secondary particles interacting further in lower altitude (higher pressure). The observed fluorescence light is the result of particles with a range of energy interacting with a "target" varying in density (pressure) and possibly composition. Improved understanding of these dependencies helps to mitigate possible systematic uncertainties.

The experiment consisted of a cylindrical vessel mounted coaxial on the beam line. ${ }^{5}$ Thin windows isolated the vessel from the beam vacuum. A gas system allowed gas of various composition (dry air and nitrogen) to flow with a pressure between 3 and 760 Torr. The fluorescence light was detected by two PMT's, orthogonal to the beam line, after passing through a UV bandpass filter similar to those used in the HiRes experiment. After various corrections, the measured yield, in units of photons per beam electron per meter, was plotted against pressure. The resulting yield curve was fitted to the function $Y(p)=\frac{C}{1 / p+1 / p^{\prime}}$ where $C$ and $p^{\prime}$ are free parameters. The results for dry air and nitrogen at $1 \mathrm{~atm}$ are: $Y_{\text {air }}=4.42 \pm 0.73$ and $Y_{N_{2}}=29.2 \pm 4.8$ photons per electron per meter. These values are consistent with existing data.

Furthermore, yield curves were obtained for four different air-nitrogen mixtures at $25 \%, 40 \%, 50 \%$, and $75 \%$ dry air. These could be useful in a detailed model of atmospheric effects. The yield measured at an energy of $28.5 \mathrm{GeV}$ also provides a unique data point when combined with earlier low energy data. The result agrees with calculation of energy deposits by charged particles in air.

\subsubsection{Spectrally Resolved Air Fluorescence Yield}

The fluorescence light may be detected at large distances, even beyond $30 \mathrm{~km}$, within HiRes' sensitive range of 300-410 nm. Because atmospheric attenuation has 
a non-linear wavelength dependence, uncertainty in the fluorescence spectrum could lead to a large error in energy determination. For example, Rayleigh scattering has a $1 / \lambda^{4}$ dependence. An error of $40 \%$ at the $390 \mathrm{~nm}$ line intensity results in a $25 \%$ error in the event energy. In order to correct for this effect, the fluorescence spectrum must be measured accurately.

The measurement setup was similar to that discussed in the previous section, except for the addition of a filter wheel. Fluorescence yields were measured at each of the 12 narrow-band filters $(296-425 \mathrm{~nm}) .{ }^{6}$ As a cross-check, an independent spectrograph system was used to measure the spectral shape. Data were collected at a pressure range of 5-750 Torr, for pure nitrogen, dry air, as well as local humid air.

The measured spectrum agrees well with the reference spectral shape used in HiRes, indicating that only small corrections are required.

\subsubsection{Energy Dependence of Air Fluorescence Yield in an EM Shower}

The electromagnetic shower initiated by a UHECR may be viewed as a superposition of many sub-showers. As the shower develops, a large flux of low energy Bremsstrahlung photons and electron-positron pairs are produced. The charged particles transfer energy to atmospheric molecules via the usual $\mathrm{dE} / \mathrm{dx}$ mechanism which is strongly energy dependent. The molecules, in turn, lose energy via fluorescence or by collisional processes. The connection between the observed fluorescence yield and cosmic ray energy is thus indirect and energy dependent. It is necessary to verify the assumption that the total fluorescence light in an air-shower is proportional to the number of shower charge particles.

The FLASH experiment made use of actual electromagnetic showers produced by an accelerator particle beam at $28.5 \mathrm{GeV}$ interacting with an alumina target. ${ }^{7}$ The thickness of the target was varied to allow measurement of total charged particle and fluorescence yields as a function of shower development age (depth). The agreement in the resulting fluorescence and ionization longitudinal shower profiles confirms the validity of the fluorescence technique.

\section{Elucidate Acceleration Principles in the Laboratory}

The acceleration mechanism that produced the UHECRs continues to be a topic of active research. In stochastic models, the particles gain energy by scattering among the magnetic fields created by magnetohydrodynamic shocks. In the following, however, we discuss models where collective plasma effects power particle acceleration.

\subsection{Inductive and Electrostatic Acceleration}

Relativistic jets are commonly observed in astrophysical sources such as active galactic nuclei and gamma-ray bursts. They are a key element in many models of cosmic acceleration. There have been many simulation studies to understand their dynamics. In a recent work, rapid particle acceleration was observed in particle-in- 
cell simulations of relativistic jet-plasma interactions. ${ }^{15}$ The evolution of a narrow, charge-neutral, electron-position jet propagating through a stationary, unmagnetized electron-ion plasma was investigated in detail. This geometry made possible exploration of physics occurring on the plasma wavelength scale.

The interaction excited magnetic filamentation, as well as electrostatic plasma instabilities. The electron and positron filaments tend to separate from each other because of the Lorentz force between them. While the electron filaments are confined by the heavier-mass background plasma ions, the positron filaments are expelled from the jet interior. As the positron filaments move away from the jet and from each other, the azimuthal magnetic field associated with the filaments decreases rapidly. This induces a large and positive longitudinal electric field. Once separated, these charge filaments also excite longitudinal electrostatic oscillations in the background plasma. The resulting acceleration increased the longitudinal momentum of about half the positrons by $50 \%$ with a maximum gain exceeding a factor of 2 .

This is a very robust process that takes place over a large range of simulation parameters, as long as conditions for Weibel instability are satisfied. This mechanism could account for the observed gamma rays and provide an injection mechanism for other ultra high energy acceleration models.

\subsection{Alfven Shock Wakefield Acceleration}

Ultra high energy cosmic ray events exceeding the GZK cutoff of $10^{19} \mathrm{eV}$ have been found in recent years. This has imposed an acute challenge to find out their sources as well as their acceleration mechanism, if they are indeed ordinary particles such as protons. The existing paradigm for cosmic acceleration, namely the Fermi mechanism, as well as its variants, such as the diffusive shock acceleration, are not effective in reaching ultra high energy. To address this issue, Chen, Tajima and Takahashi proposed a new cosmic acceleration mechanism based on the plasma wakefield excited by the astrophysically abundant Alfven shocks in a relativistic plasma outflow ${ }^{16}$. Based on the experience gained over the past 20 years on plasma acceleration studies, the authors suggest that the plasma wakefield so excited by the Alfven shocks can provide huge acceleration gradients while the particles so accelerated would suffer little collisional and radiational energy losses.

Similar to that driven by lasers or particle beams, the plasma wakefield excited by an Alfven shock is induced through the ponderomotive potential of the Alfven pulse, which also carries with it a mutually perpendicular $E$ and $B$ fields that are both transverse to its direction of propagation. The difference is that unlike the fields of the laser or particle beam where the magnitudes of $E$ and $B$ are comparable, for Alfven waves $E / B \sim v_{A} / c$, where $v_{A}$ is the speed of the Alfven wave and typically $v_{A} \ll c$. The situation is different, however, when the the plasma that supports the Alfven shocks or waves has a bulk relativistic flow. In that case $v_{A} \sim c$ and the wakefield excitation becomes very analogous to that by lasers and particle beams. It is under such astrophysical setting that the Alfven plasma wakefield can 
become effective in accelerating cosmic particles to ultra high energies. It has been generally agreed by experts that such relativistic plasma outflow or jets are indeed astrophysically abundant, for example from gamma ray bursts or Active Galactic Nuclei.

Simulation efforts using one-dimensional particle-in-cell code have been under way during the past several years. ${ }^{17}$ Recent results ${ }^{18}$ indicate that a plasma wakefield can indeed be excited by an Alfven shock propagating in a stationary background plasma. Since the trapped plasma electrons quickly gain energy and the wakefield propagates with a speed much less than the speed of light, the phaseslippage rapidly breaks the waves. Nevertheless, it accomplishes an important first step, i.e., that the Alfven-induced plasma wakefield does exist. An important next step in simulations is to introduce a relativistic bulk flow to the background plasma, so as to witness the sustained energy gain by the test trapped particles.

\section{Prospects of Future LabAstro Experiments}

In previous sections, we discussed the possibility that the astrophysical jet's kinetic energy is converted into plasma instabilities which in turn power particle acceleration and radiation. While the extreme astrophysical conditions cannot fully be replicated in the laboratory, our aspiration and challenge is to devise and perform experiments that will uncover the physical principles involved that can be scaled up to understand these powerful astronomical sources.

At $\mathrm{SABER}^{9}$, relativistic electron-positron plasma "jets" can be created by a high-energy-density beam showering in a solid target. The dynamics of jet-plasma interaction can be investigated over a scale length of tens of collisionless skin-depths. In a collisionless plasma, the mean-free-path is long compared to the skin depth so the latter is the relevant scale. Current simulation techniques can accurately resolve the physics on this scale. Laboratory results can thus be applied to astronomical collisionless plasmas and provide important tests of our ability to simulate these effects.

Some of the important questions we will address are the following. Will magnetic filamentation occur, and cause inductive and wakefield acceleration? Will the radiation spectrum confirm that observed in astrophysical sources? What are the damping and saturation mechanisms that allow astrophysical jets to survive these violent plasma instabilities and propagate long distances? Magnetic fields probably contribute to this stability and will be addressed by imposing a background field of varying strength and configuration. The effects of radiation damping will be studied as well. Can an Alfven shock propagating in a relativistic plasma excite wakefields, and accelerate particles to ultra high energy? A two-stage experiment will test whether particles accelerated as a result of magnetic filamentation will be able to seed ultra high energy acceleration in the Alfven shock wakefield. 


\section{Summary and Outlook}

High energy accelerator beams have proven to be valuable tools in the investigation of ultra high energy cosmic rays. Experiments carried out at the SLAC FFTB have been able to shed light on some of the pressing issues in UHECR observations. Simulation and theoretical work on the physical mechanisms producing UHECR have led to novel ideas that may be tested at future accelerator facilities. SABER, a new accelerator facility proposed at SLAC, will provide beams with unprecedented high-energy-density. It will make possible the investigation of cosmic acceleration mechanisms and other high-energy-density astrophysical phenomena in a unique terrestrial laboratory.

\section{Acknowledgements}

We would like to thank the conference organizers for their kind hopitality. We would also like to thank R. Noble, K. Reil, R. Bingham, R. Sydora, P.H-W. Hwang, and G-L. Lin for many fruitful discussions.

\section{References}

1. National Research Council, Frontiers in High Energy Density Physics, The National Academies Pres, Washington, D.C., 2003.

2. P. Chen, AAPPS Bull. 133 (2003); astro-ph/0303350.

3. J.S.T. Ng, in Proceedings of the $28^{\text {th }}$ ICFA Advanced Beam Dynamics Workshop, Higashi Hiroshima, Japan (SLAC-PUB-10134, 2003).

4. D.Saltzberg et al., Phys. Rev. Lett. 86, 2802 (2001).

5. J. Belz et al., Astropart. Phys. 25, 129 (2006).

6. K. Reil et al., in Proceedings of $22^{\text {nd }}$ Texas Symposium, Stanford, California (SLACPUB-11068, 2004).

7. J. Belz et al., Astropart. Phys. 25, 57 (2006).

8. P. Gorham et al., "Askaryan Effect in Ice: SLAC Calibration of the ANITA Experiment", March 2006 (unpublished); for a review of the ANITA experiment, see P. Miocinovic et al., in Proceedings of $22^{\text {nd }}$ Texas Symposium, Stanford, California (SLACPUB-11068, 2004); astro-ph/0503304.

9. SABER: The South Arc Beam Experimental Region Project, SLAC, Dec. 2005, (unpublished). http://www.slac.stanford.edu/grp/rd/epac/LOI/SABER.pdf

10. M. Takeda et al., Astrophys. J. 522, 225 (1999).

11. D.J. Bird et al. Astrophys. J. 441, 144 (1995).

12. J.N. Mathews, in Proceedings of the $28^{\text {th }}$ ICFA Advanced Beam Dynamics Workshop, Higashi Hiroshima, Japan, 2003.

13. K. Greisen, Phys. Rev. Lett. 16, 748 (1966); G.T. Zatsepin and V.A. Kuzmin, Zh. Eksp. Teor. Fiz. 4, 114 (1966) [JETP Lett. 4, 78 (1966).]

14. See reports to the International Workshop on Fluorescence Measurements, 2005, at $<$ http://lappagenda.in2p3.fr/cdsagenda/fullAgenda.php?ida=a04141>.

15. J.S.T. Ng and R.J. Noble, Phys. Rev. Lett. 96, 115006 (2006).

16. P. Chen, T. Tajima, and Y. Takahashi, Phys. Rev. Lett. 89, 161101 (2002).

17. K. Reil, R. Sydora, A. Spitkovsky and P. Chen, in 31st EPS Conf. Plasma Physics, London, EAC 28G, P-1.006 (2004).

18. K. Reil, P.Chen and R. Sydora, in preparation (2006). 